\title{
Charactéristiques d'une population introduit du Grand brochet, Esox lucius, dans le lac Ramsay, Parc de la Gatineau, Québec, et impact sur l'ichtyofaune
}

\author{
Josiane VAChON ${ }^{1}$, Brigitte F. LaValléE, et François Chapleau
}

Département de biologie, Université d'Ottawa, Casier Postal 450, Succursale A, Ottawa, Ontario K1N 6N5 Canada

${ }^{1}$ Auteur correspondant: jvach026@alumni.uottawa.ca

Vachon, Josiane, Brigitte F. Lavallée, et François Chapleau. 2005. Charactéristiques d'une population introduit du Grand brochet, Esox lucius, dans le lac Ramsay, Parc de la Gatineau, Québec, et impact sur l'ichtyofaune. Canadian FieldNaturalist 119(3): 359-366.

En 1995, la présence du Grand brochet (Esox lucius), une espèce piscivore, a été notée pour la première fois dans le lac Ramsay, Parc de la Gatineau (Québec). Il a été déterminé que l'espèce a été introduite après l'été 1991. Un échantillonnage exhaustif de l'ichtyofaune du lac en 2001 et 2002 a permis de constater que trois des 17 petites espèces de poissons du lac sont probablement disparues: le Mulet perlé (Margariscus margarita), l'Épinoche à cinq épines (Culaea inconstans) et une forme rare de l'Épinoche à trois épines (Gasterosteus aculeatus). Il est prédit que plusieurs autres espèces pourraient disparaître au cours des prochaines années. La croissance du Grand brochet dans le lac Ramsay est comparable aux autres lacs du sud du Québec. En 2001, le rapport femelles:mâles était de $15: 1$, une valeur anormale et inexpliquée.

Mots clés: Esox lucius, Grand brochet, introduit, piscivore, croissance, petits lacs, Parc de la Gatineau.

In 1995, the Northern Pike (Esox lucius), a piscivorous fish, was captured for the first time in Ramsay Lake, a small lake of the Gatineau Park (Québec). It was determined that this species was introduced after the summer of 1991. An exhaustive survey of the ichthyofauna of this lake in 2001 and 2002 indicated that three of the 17 small-bodied species of the lake have probably disappeared: the Pearl Dace (Margariscus margarita), the Fivespine Stickleback (Culaea inconstans) and a special form of the Threespine Stickleback (Gasterosteus aculeatus). It is predicted that several other species may be extirpated in the next few years. Northern Pike growth in the lake is comparable to other values obtained for other southern Québec lakes. In 2001, the female:male ratio was highly unbalanced and difficult to explain at $15: 1$.

Key Words: Esox lucius, Northern Pike, introduction, piscivory, growth, small lakes, Gatineau Park.

L'introduction d'espèces exotiques de poissons perturbe les communautés animales aquatiques (He et Kitchell 1990; Chapleau et al. 1997; Knapp et Matthews 2000; Economidis et al. 2000; Rahel 2000; Huckins et al. 2000; Findlay et al. 2000). Les petits lacs nordiques sans poissons piscivores de l'Amérique du nord comptent beaucoup moins de petites espèces de poissons que les lacs avec piscivores (Tonn et Magnuson 1982; Robinson et Tonn 1989; Tonn et al. 1992). De plus, l'introduction d'une espèce piscivore dans un lac sans piscivore entraîne inexorablement la disparition de plusieurs petites espèces (Chapleau et al. 1997; Whittier et al. 1997; Whittier et Kincaid 1999; Findlay et al. 2000). Ces espèces sont éliminées des petits lacs parce que toutes les classes d'âge sont vulnérables à la prédation et qu'elles ne peuvent pas trouver un refuge contre l'action prédatrice des poissons piscivores (Tonn et al. 1992). Le cadre temporel du processus d'extinction locale suite à l'introduction de piscivores n'a été que peu étudié (voir Demers et al. 2001a and 2001b). En effet, la plupart des études n'ont documenté que le fait accompli, souvent plusieurs décennies après l'introduction initiale des piscivores (Chapleau et al. 1997; Whittier et Kincaid 1999; Findlay et al. 2000).
Le lac Ramsay est un lac de 10,3 hectares qui a été inclus dans une étude sur l'impact de l'introduction (vers 1908) d'espèces piscivores sur les communautés de poissons des petits lacs du Parc de la Gatineau (Chapleau et al. 1997). En 1991, ce lac sans piscivores contenait 17 espèces dont 12 espèces de petite taille (incluant 10 cyprinidés). De fait, ce lac présentait l'ichtyofaune la plus diversifiée des petits lacs (moins de 60 hectares) du Parc de la Gatineau. À l'automne 1995, des spécimens d'une espèce piscivore, le Grand brochet (Esox lucius), ont été capturés dans le lac Ramsay. Le plus vieux spécimen étant âgé de cinq ans, nous constatons donc qu'une introduction non-autorisée de Grands brochets a été faite après l'été 1991. Depuis, des échantillonnages (1998, 1999, 2001 et 2002) ont révélé que le Grand brochet était bien établi dans le lac Ramsay.

Le premier objectif de notre étude est de déterminer si l'introduction d'une espèce piscivore après l'été 1991 a eu un impact sur le nombre d'espèces de poissons de petite taille dans le lac Ramsay. Un échantillonnage intensif des espèces du lac en 2001 et 2002 a permis de répondre à cette question. Le deuxième objectif de notre étude est d'examiner les paramètres biologiques de la population de Grand brochet (croissance, coeffi- 
cient de condition, ratio des sexes) dans le lac Ramsay. Il est postulé que le Grand brochet montrera une croissance particulièrement rapide dans ce lac du fait qu'il se retrouve dans un habitat qui possède une grande abondance de petits poissons et aucun autre piscivore.

\section{Matériel et méthode}

Le lac Ramsay $\left(45^{\circ} 35^{\prime} 54^{\prime \prime} \mathrm{N}, 76^{\circ} 06^{\prime} 00^{\prime \prime} \mathrm{O}\right.$; profondeur maximale : 9,5 m; profondeur moyenne : 4,1 m) est un petit lac du bouclier canadien à $200 \mathrm{~m}$ au-dessus du niveau de la mer. Son littoral est caractérisé par une abondance de plantes aquatiques mais également par des zones couvertes de sphaigne ainsi que des plages de sable et gravier. Le site est fréquenté irrégulièrement par des campeurs l'été et des pêcheurs l'été comme l'hiver (observation personnelle).

Le tableau 1 énumère les 17 espèces de poissons trouvées dans le lac en 1991 (Chapleau et al. 1997). Cette liste provient d'un échantillonnage fait en 1991 et est combinée à une liste d'espèces publiée par Rubec (1975). En 1991, le lac contenait une forme relativement rare d'Épinoche à trois épines (Gasterosteus aculeatus). Il s'agit d'une forme habituellement anadrome qui présente une série de plaques osseuses le long du corps et qui a pénétré dans la région en même temps que la mer Champlain (McAllister et Coad 1974). Elle est également restée prisonnière des lacs Hawley, Kidder, Pink et Canard suite au retrait des glaciers (Wisconsin) (Rubec 1975).

Des échantillonnages exhaustifs de la communauté de poissons du lac Ramsay ont été faits en 1971 (Rubec, 1975), 1991 (Chapleau et al. 1997), 2001 et 2002. En 1995 et 1998, l'échantillonnage était surtout axé vers la capture de Grands brochets. En 1995, des Grands brochets furent capturés au filet maillant dans le cadre d'un cours de biologie (Université d'Ottawa). Les mesures ainsi que les cleithra des poissons ont été conservés. En 1998, les engins de pêche comprenaient: trois filets maillants expérimentaux $(50 \mathrm{~m} \times 1,8 \mathrm{~m}$ avec mailles variant entre 2,5 et $15 \mathrm{~cm}$ ) et un filet trémail $(37 \times 1.2 \mathrm{~m}$; panneau extérieur avec mailles de $20 \mathrm{~cm}$ et panneau intérieur avec mailles de $2,5 \mathrm{~cm}$ ) et deux verveux (aile de 9,1 $\mathrm{m} \times 0,9 \mathrm{~m}$ avec anneaux de $76 \mathrm{~cm}$ de diamètre, mailles de $6 \mathrm{~mm}$ ). En février 1999 , 20 brimballes ont été utilisées pendant deux jours ( 8 heures par jour). Elles étaient réparties également dans les deux sites où les captures ont été les plus nombreuses l'automne précédant. Un seul Grand brochet a été capturé lors de la pêche hivernale. Ce spécimen n'a pas été inclus dans les analyses. En 2001, nous avons utilisé les mêmes filets qu'en 1998 et la période de pêche active a été de 72 heures ( 24 heures du 19 au 21 septembre et 48 heures du 27 au 29 septembre). Tous les Grands brochets capturés ont été sacrifiés, rapportés au laboratoire dans des glacières et congelés. Les spécimens d'autres espèces capturées dans les filets (Barbotte brune, Ameiurus nebulosus, et Meunier noir, Catostomus commersoni) ont été remis à l'eau. L'échantillonnage des poissons de petite taille (surtout des cyprinidés) en 2001 a été fait en utilisant une seine à bâtons de $10 \mathrm{~m}$ de longueur et deux verveux (diamètre de l'ouverture: $76 \mathrm{~cm}$; ailes $9 \mathrm{~m}$ ). En 2002, seulement la seine a été utilisée. Les poissons de petite taille ont été remis à l'eau sauf pour quelques spécimens qui ont été conservés pour la confirmation d'identification en laboratoire. Un seul Grand brochet $(0+)$ a été capturé et il n'est pas inclus dans les analyses. L'effort d'échantillonnage des petites espèces en 2001 et 2002 était plus élevé qu'en 1991. En 1991, nous avons échantillonné une surface de $300 \mathrm{~m}^{2}$ avec la seine alors que les verveux ont été actifs pendant 260,25 heures. En 2001 et 2002, l'échantillonnage par la seine a couvert une surface de $3043 \mathrm{~m}^{2}$ alors que les verveux ont été laissés dans l'eau pendant 70,25 heures. Il faut noter qu'aucune espèce n'a été échantillonnée uniquement par les verveux en 1991, 2001 et 2002, ce qui suggère que la seine est le meilleur engin de pêche pour la capture des petites espèces. Nous avons donc échantillonné une surface 10 fois plus grande en 2001 et 2002 qu'en 1991.

Tous les Grands brochets ont été décongelés avant d'être pesés et mesurés (longueurs totale et standard). Un examen visuel des gonades a permis d'identifier le sexe des spécimens. Le contenu stomacal a été examiné et les proies ont été identifiées lorsque cela était possible. Les deux cleithra ont été disséqués et bouillis. La détermination de l'âge a été faite en comptant les annuli sur les cleithra selon la méthode décrite par Casselman (1979). Les âges ont été confirmés par deux auteurs. Afin de faire des rétrocalculs de la longueur des individus à tous les âges, les distances entre le foyer et les annuli ainsi qu'entre le foyer et le bord des cleithra ont été mesurées.

Le coefficient de condition des populations a été déterminé en calculant la pente de la relation entre les logarithmes de la masse et de la longueur totale (Tesh 1971 et Ricker 1979 dans Wootton 1998).

\section{Résultats \\ Ichtyofaune}

En 2001 et 2002, nous avons capturé 14 espèces de poissons (sans compter le Grand brochet), soient trois de moins que lors de l'échantillonnage de la communauté de 1991 alors qu'il n'y avait pas de Grands brochets dans le lac (tableau 1). Les espèces manquantes sont le Mulet perlé (Margariscus margarita), l'Épinoche à trois épines (Gasterosteus aculeatus) et l'Épinoche à cinq épines (Culaea inconstans). Certaines espèces ont été capturées en très petit nombre : le Méné laiton (Hybognathus hankinsoni), le Ventre citron (Phoxinus neogaeus) et l'hybride entre le Ventre rouge du nord (Phoxinus eos) et le Ventre citron. Cependant, les difficultés d'identification des spécimens appartenant au complexe Phoxinus eos $\times$ Phoxinus neogaeus nous empêchent de conclure quoi que ce soit à propos de ces espèces. Les espèces les plus communes dans le lac sont la Barbotte brune (Ameiurus nebulosus), le Crapet-soleil (Lepomis gibbosus), la Chatte de l'est 
Tableau 1. Liste des espèces de poissons dans le lac Ramsay, Parc de la Gatineau, en 1991 et en 2001-2002.

\begin{tabular}{llc}
\hline \hline \multicolumn{1}{c}{ Espèces capturées lors de l'échantillonnage de 1991 } & Nom commun & $\begin{array}{c}\text { Espèces présentes } \\
\text { en 2001 et 2002) }\end{array}$ \\
\hline Nom latin & Grand brochet & $\mathbf{x}$ \\
\hline Esox lucius ${ }^{1}$ & Umbre de vase & $\mathbf{x}$ \\
Umbra limi & Méné laiton & $\mathbf{x}$ \\
Hybognathus hankinsoni & Chatte de l'Est & $\mathbf{x}$ \\
Notemigonus crysoleucas & Méné à nageoires rouges & $\mathbf{x}$ \\
Luxilus cornutus & Museau noir & $\mathbf{x}$ \\
Notropis heterolepis & Ventre rouge du nord & $\mathbf{x}$ \\
Phoxinus eos & Ventre citron & $\mathbf{x}$ \\
Phoxinus neogaeus ${ }^{2}$ & Ventre-pourri & $\mathbf{x}$ \\
Pimephales notatus & Tête-de-boule & $\mathbf{x}$ \\
Pimephales promelas & Mulet à cornes & $\mathbf{x}$ \\
Semotilus atromaculatus & Mulet perlé & $\mathbf{x}$ \\
Margariscus margarita & Meunier noir & $\mathbf{x}$ \\
Catostomus commersoni & Barbotte brune & $\mathbf{x}$ \\
Ameiurus nebulosus & Crapet-soleil & $\mathbf{x}$ \\
Lepomis gibbosus & Dard à ventre jaune & \\
Etheostoma exile & Épinoche à cinq épines & \\
Culaea inconstans & Épinoche à trois épines & \\
Gasterosteus aculeatus & & \\
\hline \hline
\end{tabular}

${ }^{1}$ Esox lucius n'a pas été capturé en 1991.

${ }^{2}$ S'hybride de Phoxinus eos et de Phoxinus neogaeus était également présent lors de cets échantillonnage.

(Notemigonus chrysoleucas) et le Meunier noir (Catostomus commersoni).

Parmi les Grands brochets capturés en 2001, 28\% avaient l'estomac vide, $41 \%$ avaient un contenu digéré et $31 \%$ contenaient des poissons ou autres animaux non digérés. Trois des Grands brochets avaient des parties d'écrevisses, un avait capturé un petit rongeur et un autre une grenouille. Parmi les poissons consommés encore identifiables, on a noté la présence de Chattes de l'est et de Museaux noirs. Ces dernières espèces ont été capturées lors de notre échantillonnage.

\section{Croissance du Grand brochet}

À l'automne 1995, 1998 et 2001, nous avons capturé respectivement 11,34 et 32 Grands brochets. Le nombre de captures par unité d'effort de pêche (ou capture/ heure de pêche active $\times$ nombre de filets) de 1998 et 2001 étaient respectivement de 0,095 et 0,099. Le nombre de Grands brochets capturés par unité d'effort (UE) a diminué lors des deux derniers jours de pêche au filet maillant en 2001. Il était de 0,15 et 0,186 Grand brochet par UE pour les deux premiers jours de pêche, puis de 0,03 Grand brochet par UE lors des deux derniers jours.

Tous les individus étaient âgés entre $0+$ et $7+$ (Tableau 2). Les cleithra dont le premier annulus n'était pas visible n'ont pas été utilisés pour le rétrocalcul de la longueur à l'âge d'un an. Les pentes des régressions entre la longueur et la masse (coefficient de condition) étaient de 3,14, 3,07 et 2,93 pour 1995, 1998 et 2001 respectivement (figure 1). Lors de la pêche hivernale de 1999, le seul Grand brochet capturé était âgé de deux ans. En juillet 2002, un Grand brochet 0+ (longueur totale $=12,4 \mathrm{~cm}$, masse totale $=9,8 \mathrm{~g}$ ) a été capturé avec la seine.
Les longueurs moyennes rétrocalculées des Grands brochets à chaque âge et à chaque année de capture sont présentées à la figure 2 . Il y a généralement une différence significative entre les longueurs moyennes rétrocalculées des Grands brochets pour chaque année de capture, sauf lorsque les spécimens étaient âgés de trois ans (ANOVA: alpha $=0,05 ; \mathrm{F}=0,528 ; \mathrm{p}=0,593$ ). À un an, toutes les longueurs moyennes étaient significativement différentes (ANOVA: alpha $=0,05 ; \mathrm{F}=$ 60,105; $\mathrm{p}<0,000)$. À deux ans, les Grands brochets capturés en 2001 avaient une longueur moyenne significativement plus élevée que les spécimens capturés en 1995 ou 1998 (ANOVA: alpha $=0,05 ; \mathrm{F}=12,912$; $\mathrm{p}<0,000 ; \mathrm{p}_{\text {Bonferroni }}$ de 1995 et $\left.1998<0,000\right)$. À quatre et cinq ans $\left(\right.$ ANOVA $_{4 a n s}$ : alpha $=0,05 ; \mathrm{F}=5,765 ; \mathrm{p}=$ 0,007 et ANOVA $_{5 \text { ans }}$ : alpha $=0,05 ; \mathrm{F}=8,058 ; \mathrm{p}=$ $0,003)$, la longueur moyenne des Grands brochets capturés en 2001 était significativement supérieure à celle des spécimens capturés en 1998 (ANOVA $_{4 a n s}$ : alpha $=0,05 ; \mathrm{p}_{\text {Bonferroni }}=0,007 ;$ ANOVA $_{5 \text { ans }}:$ alpha $=$ 0,$\left.05 ; \mathrm{p}_{\text {Bonferroni }}=0,002\right)$.

Rapport femelles : mâles $(F: M)$

En 2001, sur un total de 32 Grands brochets capturés, seulement deux étaient des mâles (rapport F:M de $15: 1)$. Ce rapport était de $1,2: 1$ en 1995 alors qu'il était à de 5,8: 1 en 1998.

\section{Discussion}

\section{Ichtyodiversité}

Le Grand brochet a été introduit dans le lac Ramsay après l'été 1991. Depuis ce temps, trois espèces de poissons (Mulet perlé, Épinoche à trois épines et Épinoche à cinq épines) sont probablement disparues du lac 
TABLEAU 2. Longueur et masse totales moyennes, minima et maxima pour chaque année d'échantillonnage et chaque classe d'âge pour les Grands brochets (Esox lucius) du Parc de la Gatineau. Le nombre de captures (femelles : mâles) est également indiqué.

\begin{tabular}{|c|c|c|c|c|c|c|}
\hline Année & $\begin{array}{l}\hat{\text { Âge }} \\
\text { (ans) }\end{array}$ & $\begin{array}{l}\text { Longueur } \\
\text { totale min - } \\
\max (\mathrm{cm})\end{array}$ & $\begin{array}{l}\text { Longueur totale } \\
\text { moyenne } \pm \\
\text { écart-type }(\mathrm{cm})\end{array}$ & $\begin{array}{c}\text { Masse } \\
\text { totale min - } \\
\max (\mathrm{g})\end{array}$ & $\begin{array}{l}\text { Masse totale } \\
\text { moyenne } \pm \\
\text { écart-type }(\mathrm{g})\end{array}$ & $\begin{array}{c}\text { Femelles } \\
\text { : Mâles }\end{array}$ \\
\hline \multirow[t]{3}{*}{1995} & $3+$ & $52.0-67.7$ & $56.7 \pm 6.8$ & $983.0-2419.0$ & $1561.5 \pm 591.4$ & $4: 2$ \\
\hline & $4+$ & $50.0-72.0$ & $61.7 \pm 10.7$ & $1006.0-2947.0$ & $1918.0 \pm 952.3$ & $2: 2$ \\
\hline & $5+1$ & - & 61.7 & - & 1939.0 & $0: 1$ \\
\hline \multirow[t]{7}{*}{1998} & $1+$ & $33.5-37.1$ & $35.4 \pm 1.8$ & $230.0-309.0$ & $272.0 \pm 39.7$ & $3: 0$ \\
\hline & $2+$ & $34.7-41.2$ & $38.1 \pm 2.2$ & $271.0-444.0$ & $353.8 \pm 55.1$ & $10: 2$ \\
\hline & $3+$ & $56.4-58.4$ & $57.4 \pm 1.4$ & $1179.0-1317.0$ & $1248.0 \pm 97.6$ & $2: 0$ \\
\hline & $4+$ & $56.1-63.3$ & $59.3 \pm 3.7$ & $1222.0-1645.0$ & $1368.3 \pm 239.7$ & $2: 1$ \\
\hline & $5+$ & $58.3-64.8$ & $61.8 \pm 2.2$ & $1330.0-1879.0$ & $1541.8 \pm 192.2$ & $8: 2$ \\
\hline & $6+$ & $56.0-58.5$ & $57.3 \pm 1.8$ & $1092.0-1423.0$ & $1257.5 \pm 234.0$ & $2: 0$ \\
\hline & $7+$ & $66.5-71.1$ & $68.8 \pm 3.2$ & $1890.0-2427.0$ & $2158.5 \pm 379.7$ & $2: 0$ \\
\hline 1999 & $3+1$ & - & 49.5 & - & 786 & $0: 1$ \\
\hline \multirow[t]{5}{*}{2001} & $1+1$ & - & 41.5 & - & 427.0 & $1: 0$ \\
\hline & $2+$ & $42.4-50.5$ & $46.2 \pm 2.3$ & $445.0-871.0$ & $605.6 \pm 115.1$ & $13: 1$ \\
\hline & $3+$ & $51.0-54.0$ & $52.2 \pm 1.3$ & $807.0-1014.0$ & $883.0 \pm 97.3$ & $3: 1$ \\
\hline & $4+$ & $58.5-68.9$ & $64.5 \pm 4.5$ & $873.0-2213.0$ & $1502.0 \pm 546.9$ & $5: 0$ \\
\hline & $5+$ & $64.2-71.7$ & $67.3 \pm 2.8$ & $1549.0-2308.0$ & $1862.9 \pm 288.1$ & $8: 0$ \\
\hline 2002 & $0+{ }^{1}$ & - & 12.4 & - & 9.8 & $1 \mathrm{n} / \mathrm{d}$ \\
\hline
\end{tabular}

${ }^{1}$ Ces classes d'âge n'ont qu'un seul poisson.

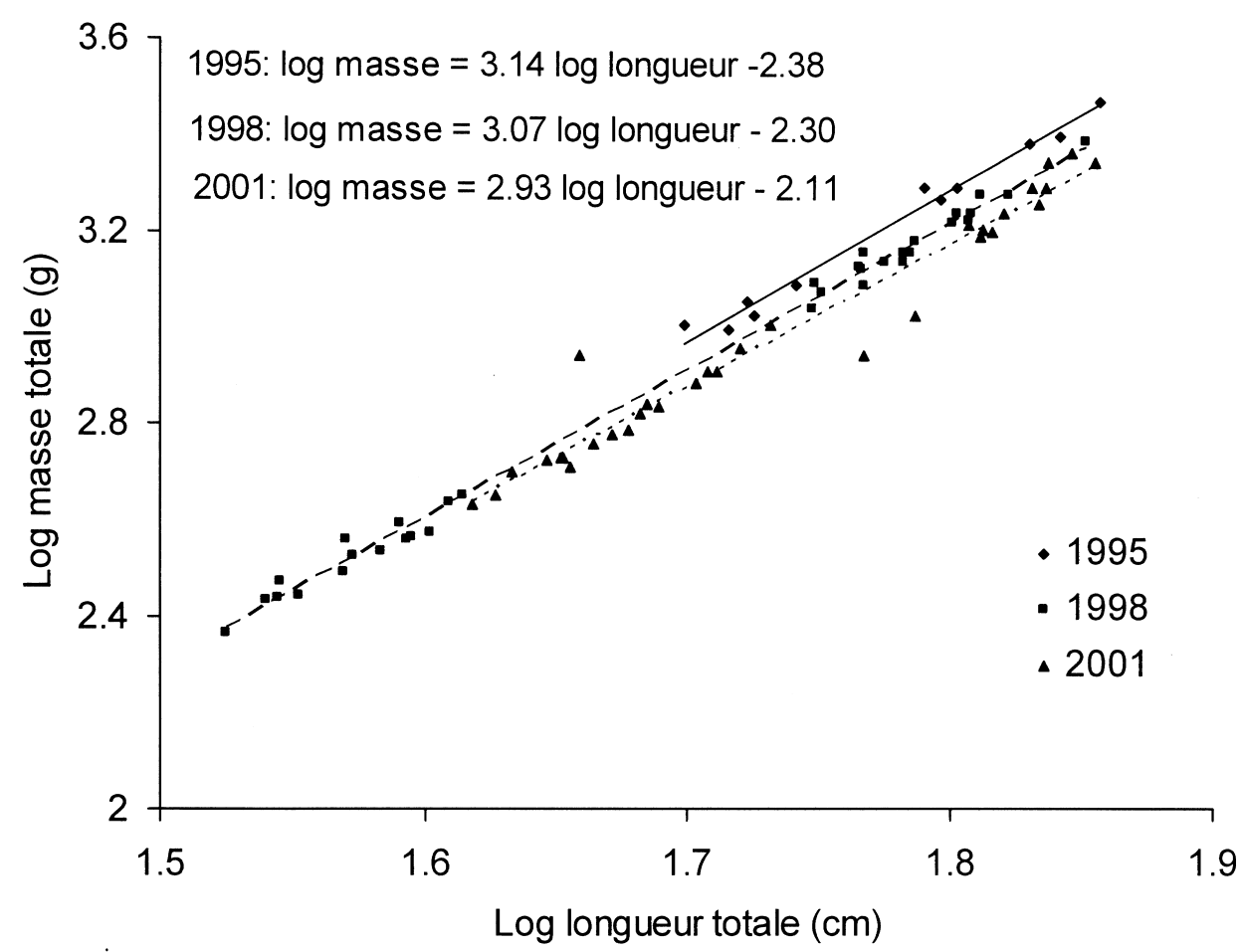

FIGURE 1. Relation logarithmique entre la masse totale et la longueur totale des Grands brochets du lac Ramsay. Le coefficient de condition correspond à la pente des droites. $1995: \mathrm{n}=11 ; 1998: \mathrm{n}=34 ; 2001: \mathrm{n}=32$. 


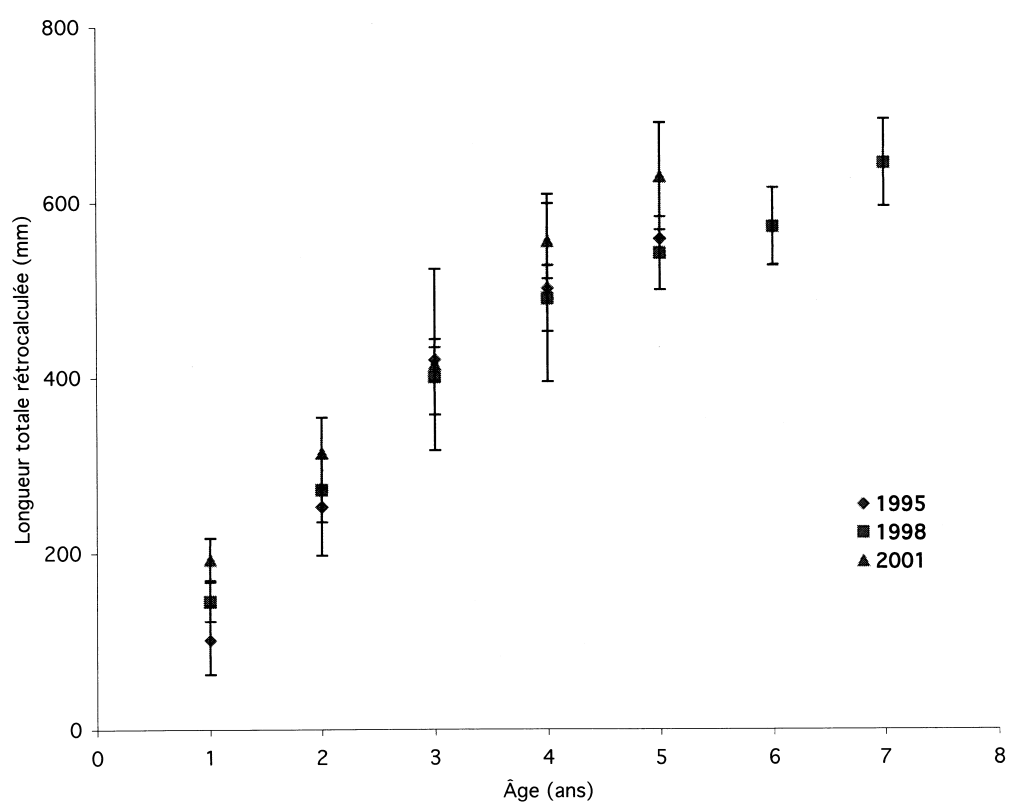

FIGURE 2. Longueur rétrocalculée à chaque âge des Grands brochets du lac Ramsay lors des années d'échantillonnage (1995, 1998, 2001). Les barres d'erreurs représentent les écarts-types.

Ramsay. Leur présence dans le lac est possible mais dans ce cas, elle reste indétectable même suite à un effort d'échantillonnage plus intensif en 2001 et 2002 qu'en 1991. Il est probable que la cause de l'absence de ces espèces soit l'introduction de l'espèce piscivore. Le processus d'extinction des petites espèces est donc bel et bien enclenché dans le lac Ramsay et il semble rapide. Nous pouvons d'ores et déjà prédire plusieurs autres extinctions au cours des prochaines années si la population de Grands brochets persiste. Chapleau et al. (1997) ont trouvé que les petits lacs du parc de la Gatineau avec piscivores introduits ont moins de la moitié du nombre d'espèces de petite taille que les lacs qui n'ont pas de piscivores. Demers et al. (2001a) ont observé que le nombre d'espèces non piscivores est passé de sept espèces en 1991 à quatre en 1997 suite à l'introduction, dans un petit lac, de deux espèces piscivores, l'Achigan à grande bouche (Micropterus salmoides) et l'Achigan à petite bouche (Micropterus dolomieu). Findlay et al. (2000) ont montré que le nombre d'espèces de cyprinidés des lacs des Adirondacks était trois fois plus faible dans les lacs ayant des piscivores introduits. Nous pouvons donc prédire que la faune actuelle du lac Ramsay pourrait passer de 12 à quatre ou six petites espèces au cours des prochaines années.

D'ailleurs, les trois espèces disparues appartiennent à la liste des cinq espèces présentes exclusivement dans les lacs sans espèces piscivores du Parc de la Gatineau (Chapleau et al. 1997). Les deux autres espèces de cette liste, l'Umbre de vase (Umbra limi), et le Méné laiton (Hybognatus hankinsoni) sont donc les prochaines candidates logiques à l'extinction locale. D'ailleurs, cette dernière espèce n'a été capturée que très rarement lors de notre échantillonnage alors qu'elle était abondante en 1991 (Chapleau, observation personnelle). Le fait que les quatre espèces non piscivores les plus abondantes dans le lac en 2001 et 2002 (Notemigonus chrysoleucas, Catostomus commersoni, Ameiurus nebulosus et Lepomis gibbosus) sont des espèces de taille intermédiaire démontre qu'il y a eu un changement de l'abondance relative des espèces, et cela en faveur des espèces de taille intermédiaire (Chapleau, observation personnelle).

Demers et al. (2001a) ont démontré, suite à une manipulation des communautés de poissons dans deux petits lacs du sud de l'Ontario, que les stocks de poissons diminuaient fortement (de $32 \mathrm{~kg} \mathrm{ha}^{-1} \mathrm{an}^{-1}$ à $5 \mathrm{~kg}$ $\mathrm{ha}^{-1} \mathrm{an}^{-1}$ ) en trois à cinq ans suite à l'introduction de piscivores. Dans un autre lac, ils ont également noté un certain rétablissement des stocks (de $12 \mathrm{~kg} \mathrm{ha}^{-1}$ $\mathrm{an}^{-1}$ à $32 \mathrm{~kg} \mathrm{ha}^{-1} \mathrm{an}^{-1}$ ) en trois à cinq ans suite à l'enlèvement d'une partie des prédateurs. Dans ce même lac, ils ont observé que deux espèces saisonnières sont devenues résidentes suite au retrait des Achigans à grande bouche et des Achigans à petite bouche. Il serait donc possible de penser que le prélèvement d'une partie importante de la population de Grands brochets en 1998 et en 2001 ait ralenti le processus d'extinction et ait permis à certaines petites espèces rares de se maintenir. 
De plus, en 1991, le lac Hawley, un petit lac (5,1 hectares; 17 espèces; 12 espèces de petite taille) tout juste en aval (moins de $1 \mathrm{~km}$ ) du lac Ramsay, ne comptait pas d'espèces piscivores. Lors d'un échantillonnage préliminaire en 1998, le Grand brochet a été signalé pour la première fois dans ce lac (Chapleau, observation personnelle). Donc, non seulement le Grand brochet est bien établi dans le lac Ramsay, mais il se répand présentement dans les lacs adjacents.

\section{Croissance du Grand brochet}

Les longueurs totales rétrocalculées des individus du lac Ramsay capturés en 1998 et 2001 sont comparables aux moyennes pour les populations du sud du Québec (Vallières et Fortin 1988) (voir figure 3). Les Grands brochets capturés en 1995 ont eu une croissance lente lors de leur première année.

Nous avions prévu une croissance rapide des Grands brochets au lac Ramsay à cause de l'absence d'autres espèces piscivores et de l'abondance de proies. Or, la croissance est normale. Cela peut être attribuables à une compétition intraspécifique plus importante que prévue. En effet, la petite taille du lac a probablement contribué à l'atteinte d'une densité assez élevée de Grands brochets en peu de temps. Nos résultats ne concordent donc pas avec Stocek et al. (1999) qui ont observé une croissance très rapide du Maskinongé (Esox masquinongy) suite à son introduction dans les eaux du Nouveau-Brunswick. Stocek et al. (1999) ont postulé que ce taux de croissance était dû à la faible compétition et à l'abondance de proies. Il est fort probable qu'une partie des Grands brochets capturés au lac Ramsay en 1995 soient nés dans un autre lac, ce qui expliquerait la faible longueur totale moyenne pour les individus à un et deux ans.

Dans plusieurs cas, la longueur moyenne rétrocalculée est significativement plus élevée chez les spécimens de Grands brochets capturés en 2001. On peut supposer que le nombre de grands spécimens a diminué de façon importante lors de la pêche particulièrement intensive de 1998. Les survivants à cette pêche qui ont été capturés en 2001 auraient eu une croissance particulièrement importante. Cela peut s'expliquer en partie par une diminution de la compétition intraspécifique causée par la pêche intensive.

Les Grands brochets capturés en 2001 ont un coefficient de condition de 2,93 (Figure 2) ou une croissance allométrique négative, ils sont donc plus petits par rapport à leur longueur que la normale (c'est-àdire, 3,0$)$. De plus, leur condition semble à la baisse par rapport aux années d'échantillonnage précédentes. Les valeurs pour 1995 et 1998 étaient respectivement de 3,14 et 3,07, soit une croissance allométrique positive, c'est-à-dire que les individus étaient généralement plus gros par rapport à leur longueur que la normale. Pour d'autres populations, les valeurs obtenues se situent habituellement entre 2,86 et 3,27 (Frost et Kipling 1967; Mann 1976). Il est difficile d'expliquer la diminution du coefficient de condition dans le temps pour les Grands brochets du Lac Ramsay. Il est possible que l'abondance des proies montre une diminution dans le temps, la probable extinction de trois espèces en dix ans étant un bon indicateur que la présence du Grand brochet a eu un impact sur la communauté et possiblement sur le recrutement des proies.

\section{Ratio femelles : mâles}

Le rapport femelles : mâles des Grands brochets évolue définitivement en faveur des femelles depuis 1995. Il est passé de 1,2: 1 en 1995, à 5,8: 1 en 1998, pour finalement atteindre $15: 1$ en 2001 . Ces dernières valeurs sont très élevées, mais au moins un autre résultat semblable a été observé. Frost et Kipling (1967) ont obtenu un ratio de 11,6:1 avec les Grands brochets capturés à l'âge de quatre ans en 1951 au lac Windermere (données calculées à partir des résultats de Frost et Kipling 1967). Les années précédente et suivante montrent respectivement un ratio de 2,4:1 et de 2,9:1. Après six ans, le ratio est revenu à $1: 1$. Les auteurs ne mentionnent pas ce débalancement. Dans la littérature, les proportions varient le plus souvent entre 2 : 1 et $1: 1$ (Frost et Kipling 1967 - calculé à partir des données de l'article; Casselman 1975; Mann 1976 calculé à partir des données de l'article; Massé 1979 dans Vallières et Fortin 1988; Roche et al. 1999). Casselman (1975) a par ailleurs démontré que les proportions femelles: mâles capturés des Grands brochets avaient tendance à varier selon la saison. En été (croissance somatique) et en hiver (développement des gonades), les femelles deviennent plus actives, ce qui les rend plus vulnérables à la prise au filet maillant. Or, chacun de nos échantillonnages a eu lieu vers la fin de l'été et au début de l'automne. La saison d'échantillonnage ne peut donc pas expliquer le ratio en faveur des femelles et l'évolution de ce ratio dans le temps. En septembre et octobre, les rapports femelles: mâles des populations étudiées par Casselman (1975) sont aux alentours de $1: 1$. Notre résultat suggère donc un sérieux débalancement dans la proportion des sexes dont la cause demeure inconnue.

\section{Remerciements}

Nous aimerions remercier le Parc de la Gatineau, la Commission de la Capitale Nationale et la Société de la Faune et des Parcs du Québec pour l'attribution des permis de pêche scientifique. Nous remercions également Anne-Marie Phelps, Thevia Belisle, Kevin Moon, Brent Campbell, Mélanie Barbeau, Annie Comtois, Annie-Chantal Guibord, Julie Lepage et Guy Vachon pour leur aide avec l'échantillonnage. Ce projet a pu être complété grâce à un octroi à la découverte du CRSNG à FC.

\section{Littérature Citée}

Casselman, J. M. 1975. Sex Ratios of Northern Pike, Esox lucius Linnaeus. Transactions of the American Fisheries Society 1: 60-63. 


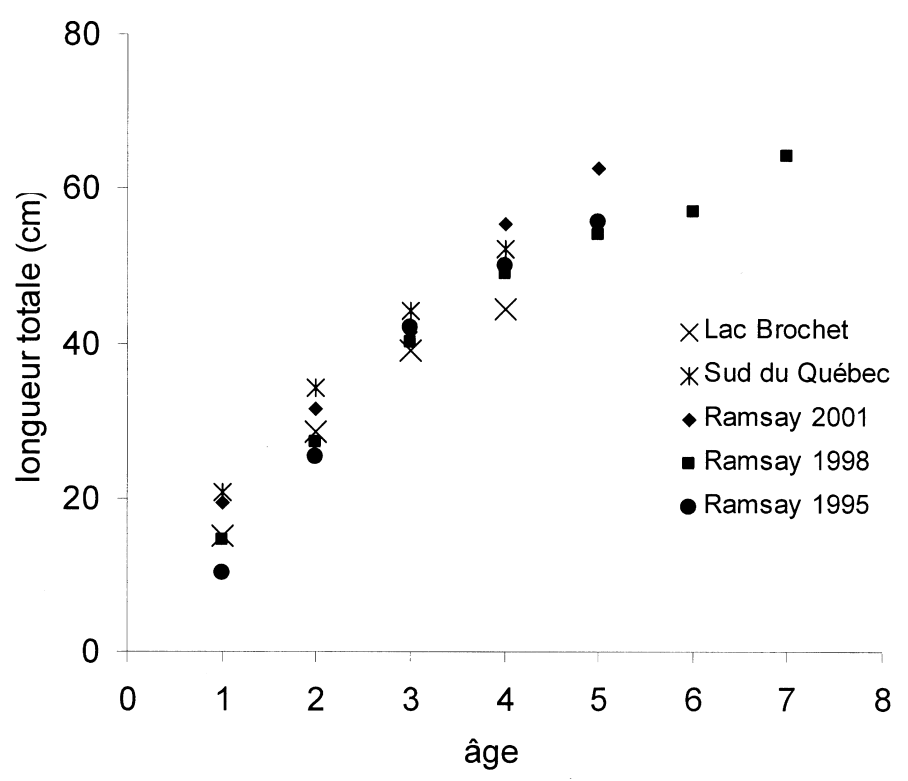

FIGURE 3. Longueur rétrocalculée à chaque âge des Grands brochets de lacs du Sud du Québec (Vallières et Fortin 1988) incluant les données du lac Ramsay pour 1995, 1998 et 2001.

Casselman, J. M. 1979. The esocid cleithrum as indicator calcified structure. Pages 249-272, édité par J. Dubé et Y. Gravel. Proceedings of the $10^{\text {th }}$ Warmwater Workshop, Special Publications. Northeast Division, American Fisheries Society. Montréal, Québec. 285 pages.

Chapleau, F., S. Findlay, et E. Szenasy. 1997. Impact of piscivorous fish introductions on fish species richness of small lakes in Gatineau Park, Québec. Écoscience 4: 259268.

Demers, E., D. J. McQueen, S. A. Popiel, A. M. Rocchi, C. W. Ramcharan, et A. Pérez-Fuentetaja. 2001a. Changes in fish community structure. Ergebnisse der Limnologie 56: 23-48.

Demers, E., D. J. McQueen, C. W. Ramcharan, et A. Pérez-Fuentetaja. 2001b. Did piscivores regulate changes in fish community structure? Ergebnisse der Limnologie 56: 49-80.

Economidis, P. S., E. Dimitriou, R. Pagoni, E. Michaloudi, et L. Natsis. 2000. Introduced and translocated fish species in the inland waters of Greece. Fisheries Management \& Ecology 7: 239-250.

Findlay, S. C., D. G. Bert, et L. Zheng. 2000. Effect of introduced piscivores on native minnow communities in Adirondack lakes. Canadian Journal of Fisheries and Aquatic Sciences 57: 570-580.

Frost, W. E., et C. Kipling. 1967. A study of reproduction, early life, weight-length relationship and growth of pike, Esox lucius L., in Windermere. Journal of Animal Ecology 36: 651-693.

He, X., et J. F. Kitchell. 1990. Direct and indirect effects of predation on a fish community: a whole-lake experiment. Transactions of the American Fisheries Society 119: 825835 .
Huckins, C. J. F., C. W. Osenberg, et G. G. Mittelbach. 2000. Species introductions and their ecological consequences: An example with congeneric sunfish. Ecological Applications 10: 612-625.

Knapp, R. A., et K. R. Matthews. 2000. Non-native fish introductions and the decline of the mountain yellow-legged frog from within protected areas. Conservation Biology 14: 428-438.

Mann, R. H. K. 1976. Observations on the age, growth, reproduction and food of the pike Esox lucius (L.) in two rivers in southern England. Journal of Fish Biology 8: 179-197.

McAllister, D. E., et B. W. Coad. 1974. Fishes of Canada's National Capital Region. Fisheries Research Board of Canada Miscellaneous Special Publication 24. 200 pages.

Rahel, F. J. 2000. Homogenization of fish faunas across the United States. Science (Washington, D.C.) 288 (5467): 854-856.

Robinson, C. L. K., et W. M. Tonn. 1989. Influence of environmental factors and piscivory in structuring fish assemblages of small Alberta lakes. Canadian Journal of Fisheries and Aquatic Sciences 46: 81-89.

Roche, W., M. O'Grady, et J. J. Bracken. 1999. Some characteristics of a pike Esox lucius L. population in an Irish reservoir. Hydrobiologia 392: 217-223.

Rubec, P. J. 1975. Fish distribution in Gatineau Park, Québec, in relation to postglacial dispersal, man's influence and eutrophication. Canadian Field-Naturalist 89: 389-399.

Stocek, R. F., P. J. Cronin, et P. D. Seymour. 1999. The muskellunge, Esox maskinongy, distribution and biology of a recent addition to the ichthyofauna of New Brunswick. Canadian Field-Naturalist 113: 230-234. 
Tonn, W. M., et J. J. Magnuson. 1982. Patterns in the species composition and richness of fish assemblages in Northern Wisconsin Lakes. Ecology 63: 1149-1166.

Tonn, W. M., C. Paszkowski, et I. Holopainen. 1992. Piscivory and recruitment: mechanisms structuring prey populations in small lakes. Ecology 73: 951-958.

Vallières, L., et R. Fortin. 1988. Le Grand brochet (Esox lucius) au Québec: biologie et gestion. Université du Québec à Montréal, pour le ministère du loisir, de la chasse et de la pêche du Québec, Direction de la gestion des espèces et des habitats, Québec. 298 pages.

Whittier, T. R., D. B. Halliwell, et S. G. Paulsen. 1997. Cyprinid distributions in Northeastern U.S.A. lakes: evi- dence of regional-scale minnow biodiversity losses. Canadian Journal of Fisheries and Aquatic Sciences 54: 15931607.

Whittier, T. R., et T. M. Kincaid. 1999. Introduced fish in Northeastern USA lakes: regional extent, dominance, and effect on native species richness. Transactions of the American Fisheries Society 128: 769-783.

Wootton, R. J. 1998. Ecology of teleost fishes. Second edition. Kluwer Academic Publishers. Fish and Fisheries Series 24. London. 386 pages.

Reçu le 8 décembre 2003

Accepté le 15 août 2005 


\section{News and Comment}

\section{Northeast Natural History Conference 2013}

The $13^{\text {th }}$ Northeast Natural History Conference (NENHC) hosted by The Association of Northeastern Biologists to be held 13-15 April 2013 at the Sheraton Springfield Hotel in Springfield, Massachusetts. This is a 3 day conference which promises to be the largest forum for researchers, natural resource managers, students, and naturalists to present current infor-

\section{American Society of Mammalogists Annual Meeting 2013}

The $93^{\text {rd }}$ annual meeting of the American Society of Mammalogists to be held 14-18 June 2013 at the Philadelphia Marriott Downtown in Philadelphia, Pennsylvania. Planned symposia include White-nose Syndrome in Bats, Ecological Niche Modeling, and

\section{Erratum The Canadian Field-Naturalist 119(3): 359}

Le titre de l'article devrait se lire : Caractéristiques d'une population introduite du Grand brochet, Esox lucius, dans le lac Ramsay, Parc de la Gatineau, Québec, et impact sur l'ichtyofaune. La bande citation de l'article devrait se lire: Vachon, Josiane, Brigitte F. Lavallée, et François Chapleau. 2005. Caractéristiques d'une population introduite du Grand brochet, Esox lucius, dans le lac Ramsay, Parc de la Gatineau, Québec, et impact sur l'ichtyofaune. Canadian Field-Naturalist 119(3): 359-366. mation on the varied aspects of applied field biology (freshwater, marine, and terrestrial) and natural history for the Northeastern United States and adjacent Canada. Registration is currently open. Deadline for abstract submissions is March 1, 2013 and early registration deadline is March 8, 2013. More information is available at http://www.eaglehill.us/NENHC_2013/NENHC2013.shtml.

Advances in Ungulate Biology. Registration is currently open. Deadline for abstract submissions is April $1^{\text {st }}$, 2013. More information is available at www.mammal society.org.

The title of the article should be : Caractéristiques d'une population introduite du Grand brochet, Esox lucius, dans le lac Ramsay, Parc de la Gatineau, Québec, et impact sur l'ichtyofaune. The citation strip should be: Vachon, Josiane, Brigitte F. Lavallée, et François Chapleau. 2005. Caractéristiques d'une population introduite du Grand brochet, Esox lucius, dans le lac Ramsay, Parc de la Gatineau, Québec, et impact sur l'ichtyofaune. Canadian Field-Naturalist 119(3): 359366. 